Article

\title{
Ecology of the River Darter in Canadian Waters: Distribution, Relative Abundance, Life-History Traits, Diet, and Habitat Characteristics
}

\author{
Thomas C. Pratt ${ }^{1}$ *, William M. Gardner ${ }^{1}$, Douglas A. Watkinson ${ }^{2}$ and Lynn D. Bouvier ${ }^{3}$ \\ 1 Fisheries and Oceans Canada, Sault Ste. Marie, ON P6A 2E5, Canada; william.gardner@dfo-mpo.gc.ca \\ 2 Fisheries and Oceans Canada, Winnipeg, MB R3T 2N6, Canada; doug.watkinson@dfo-mpo.gc.ca \\ 3 Fisheries and Oceans Canada, Burlington, ON L7S 1A1, Canada; lynn.bouvier@dfo-mpo.gc.ca \\ * Correspondence: thomas.pratt@dfo-mpo.gc.ca; Tel.: +1-705-941-2667; Fax: +1-705-941-2664
}

Academic Editor: Thilina Surasinghe

Received: 2 June 2016; Accepted: 11 October 2016; Published: 25 October 2016

\begin{abstract}
The River Darter (Percina shumardi) is a native, rarely sampled fish that has been detected in relatively small numbers since the 1930s. It has a wide North American distribution, but little is known about the species biology and distribution across the Canadian portion of its range. We revisited many historic sampling locations and sampled additional areas to (i) confirm and update the distribution of River Darters in Canadian waters; (ii) assess relative abundance; (iii) update life history characteristics; (iv) collect diet information; and (v) identify characteristics of River Darter habitat. Since 1990, a total of 1032 River Darters were recorded from 29 waterbodies across three ecoregions. River Darters were observed in relatively high abundances in both the Saskatchewan-Nelson River and Southern Hudson Bay-James Bay ecoregions. While still extant in the Great Lakes-Upper St. Lawrence ecoregion, River Darters persist at low abundance. Life history analyses indicate similar maximum age (age 4) and growth (10 mm/year) to conspecific populations in the United States, while sex ratios are generally skewed towards female dominance. River Darter populations had high flexibility in habitat use and diet, using a range of flows and depths and a variety of seasonally available prey types.
\end{abstract}

Keywords: Percina shumardi; conservation status; location; population assessment; growth; food habits

\section{Introduction}

North America darters, members of the perch family (Percidae), now consist of over 200 recognized species [1]. Many of these generally diminutive species are imperiled and have been assigned conservation statuses [2]. Given their relative rarity, little is known about the biology of many species in this group of fishes. One such poorly-studied species is the River Darter (Percina shumardi). The River Darter has a wide North American geographic distribution, ranging from Texas in the south to northern Manitoba in the north, and from the Ohio River (Pennsylvania) in the east to the Saskatchewan River (Saskatchewan) in the west [1,3,4]. Despite this wide distribution, relatively few individuals have been documented in most areas, and the River Darter is a species of conservation concern in many jurisdictions. For example, the species is considered critically imperiled in a number of states (Georgia, Kansas, Michigan, Ohio, Pennsylvania, and West Virginia), and vulnerable in the province of Ontario [5]. The River Darter has been collected haphazardly in central Canada in relatively small numbers since the 1930s, but has received little attention from researchers.

As the Committee on the Status of Endangered Wildlife in Canada (COSEWIC) was scheduled to review the conservation status of the River Darter in 2016 for possible listing under the federal Species at Risk Act, it was important to update our knowledge of this species as the latest review of the distribution and status of the River Darter in Canada was completed in 1990 and no information had 
been published since that time. The 1990 review described populations of River Darters from Manitoba, northwestern Ontario, and southern Ontario [6]. The species occurs in three geographically-isolated ecoregions, which are termed freshwater biogeographic zones by COSEWIC: the Saskatchewan-Nelson River Biogeographic Zone, the Southern Hudson Bay-James Bay Biogeographic Zone, and the Great Lakes-Upper St. Lawrence Biogeographic Zone [7]. In Manitoba, the River Darter occurred in the Assiniboine, Nelson, Red, and Winnipeg River watersheds as well as lakes Dauphin, Manitoba, Winnipegosis, and Winnipeg watersheds. In Ontario, River Darters were found in the English and Rainy river watersheds that are part of the Nelson River watershed, as well as the Attawapiskat, Albany, Severn, and Winisk river watersheds that flow into Hudson or James Bay, and the Lake St. Clair watershed in the Great Lakes drainage.

Herein we review current knowledge (post-1990) on the status and distribution of River Darters in Canada. We collated River Darter records obtained incidentally in fish surveys since 1989, and we conducted targeted surveys for River Darters at many of the historical sites while also sampling additional waterbodies within the historic known range. Our specific study objectives were to (i) provide an assessment on the distribution of the River Darter; (ii) assess its relative abundance; (iii) update life history characteristics; (iv) collect diet information; and (v) identify habitat characteristics (including depth, flow, and temperature) associated with River Darters collected along the northern portion of the species range.

\section{Materials and Methods}

\subsection{Incidental River Darter Surveys}

We collated records of River Darters incidentally captured by Fisheries and Oceans Canada (DFO) staff during trawling, seining, and electrofishing (backpack and boat) surveys between 1996 and 2014, as well as Royal Ontario Museum (ROM) fish collection records from 1990 to 2014 (Figure 1). No life history or biological data were available for fish captured during these surveys. These surveys were made across a vast geographic area, which differ greatly in their underlying climate and geology. River Darter populations in the Saskatchewan-Nelson River and Southern Hudson Bay-James Bay ecoregions primarily occur in the boreal shield region which is generally characterized by thin soils, exposed bedrock, a cool climate, and water bodies that ultimately drain into the Arctic Ocean [8]. Populations in the Great Lakes-Upper St. Lawrence ecoregion are in systems that drain into the Atlantic Ocean and encounter a more moderate climate in the mixedwood plains region of Canada, with a more productive underlying geology comprised of carbonate-rich Paleozoic bedrock [8]. There are differences in anthropogenic impacts among ecoregions as well, with the highest impacts occurring in the more densely populated Great Lakes-Upper St. Lawrence ecoregion, and the least impacts in the sparsely populated Southern Hudson Bay-James Bay ecoregion [9]. 

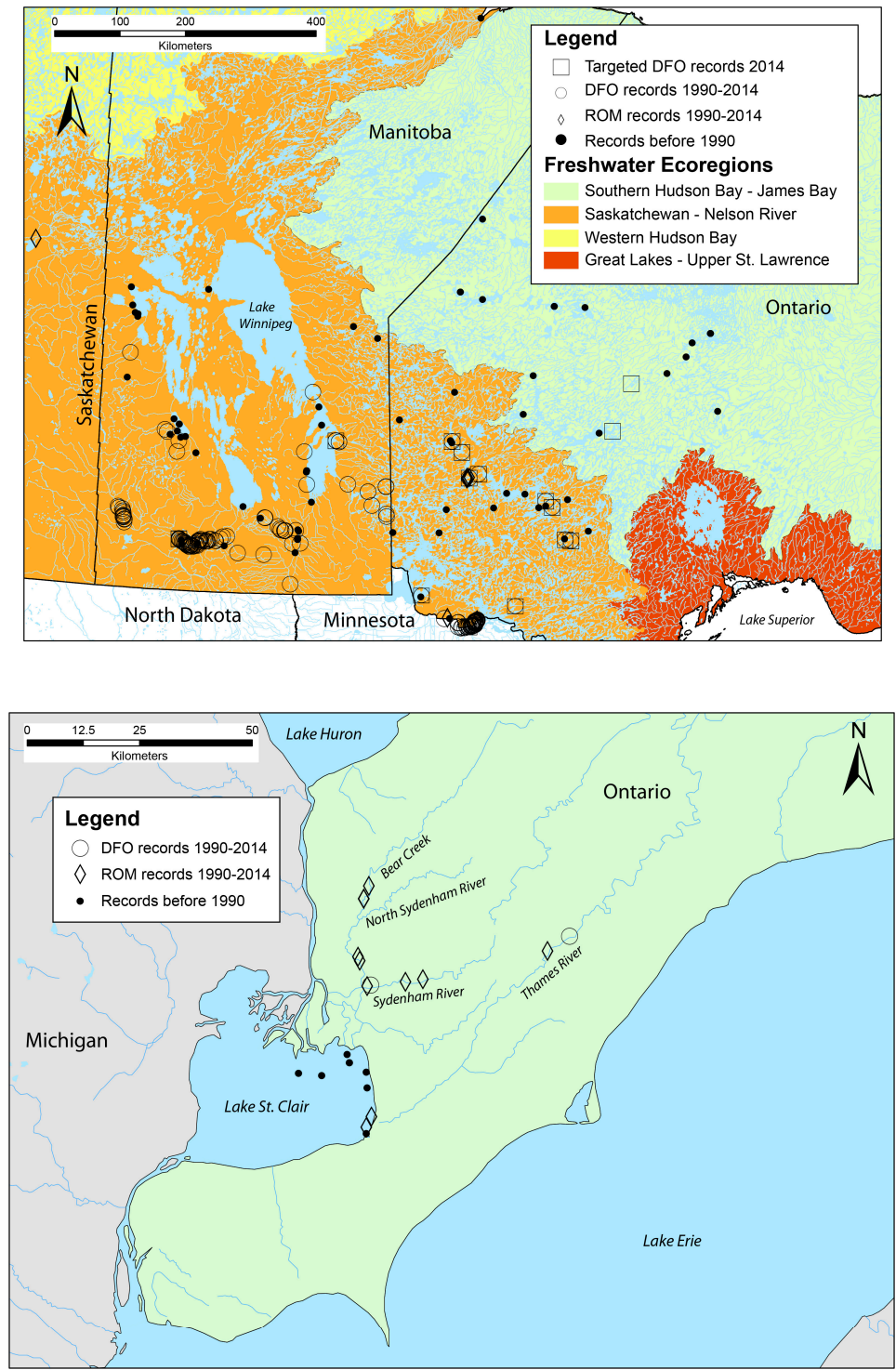

Figure 1. Locations of historic (pre-1990; solid circle), Royal Ontario Museum (ROM) (1990-2014; open diamond), Fisheries and Oceans Canada (DFO) incidental (open circle) and DFO targeted (open square) River Darter collections. The top panel displays collections from the Saskatchewan-Nelson and Southern Hudson Bay-James Bay ecozones in Manitoba and northwestern Ontario, while the lower panel displays collections in southern Ontario from the Great Lakes-Upper St. Lawrence ecozone.

In 2013, a large number of River Darters were incidentally collected during a fish community study conducted by DFO using boat electrofishing in the Rainy River, Ontario. River Darters were incidentally captured in surveys conducted in Manitoba by DFO from 1996 to 2009 with a variety of gear. Equipment that was successfully used to capture River Darters included seine nets and boat electrofishing, as well as a variety of trawls including a beam trawl, a Missouri trawl [10], and a mini-Missouri bottom trawl [11].

\subsection{Targeted River Darter Surveys}

From 2012 to 2014, we conducted targeted River Darter surveys at 18 sites in Ontario and Manitoba at locations either within historic River Darter localities or at probable areas of occupancy. Targeted sampling used a mini-Missouri bottom trawl to sample suitable areas in both lakes and rivers. The head rope of the mini-Missouri trawl is $2.44 \mathrm{~m}$ long with two floats spaced $0.61 \mathrm{~m}$ apart on center; the foot 
rope of the trawl is $3.66 \mathrm{~m}$ long. The trawl narrows from $2.44 \mathrm{~m}$ wide at the head rope to $0.46 \mathrm{~m}$ wide at the mid-section and cod end [11]. To determine whether sampling was feasible prior to trawling, the depth and substrate of a potential site was assessed using Hummingbird 1198c side-scan sonar to ensure there were no obstacles to trawling. When an area devoid of large rocks and trees nor subject to extreme changes in bathymetry was located, the trawl was deployed at the upstream end of the site, GPS coordinates were taken, and the net was pulled with a boat downstream on the bottom at a speed faster than the current. In lakes, the net was either pulled across current or with the current if the waves were higher than $20 \mathrm{~cm}$. At the end of the trawl, GPS coordinates were recorded so a trawl distance could be determined and the net was lifted. Captured fishes were placed into a cooler filled with fresh lake water, identified to species and all by-catch released. Approximately 30 River Darters from each sampling site were retained for further analysis. At most sites, multiple trawls were conducted to ensure sufficient River Darters were obtained. Retained fish were preserved in $70 \%$ ethanol. At the northwestern Ontario sites sampled in 2014, basic water quality data (temperature, $\mathrm{pH}$, turbidity, and dissolved oxygen) were collected using a YSI 6820V2-M multi-parameter water quality sonde.

\subsection{Laboratory Analysis}

For each captured River Darter, length and weight were measured. One or two specimens from most sites were then sent to the Royal Ontario Museum for identification validation and archiving. For the remaining fish, gender and maturity status (from dissected gonads; mature, immature, and unknown) were recorded, and stomachs were dissected and gut contents examined to the family level, or to order if family identification features were unidentifiable.

Sagittal otoliths were removed from each fish for age and growth assessment. As initial readings of otoliths for age interpretation were difficult, otoliths were stained, embedded in epoxy, and the convex surface was ground along the sagittal plane and polished to enhance the resolution of annular rings [12]. Two agers, working independently, viewed the digital photos and assigned ages to each photo. In situations where the agers did not agree on the ages, a third ager examined the photos and assigned an age.

\subsection{Data Analysis}

For each location, River Darter density (number/ha) was calculated by assuming trawls and boat electrofishers had an effective sampling width of $2.5 \mathrm{~m}$. Data from mini-Missouri trawls, which were fished among all three ecoregions, were used to examine for differences in mean density among ecoregions. As there were concerns about spatial autocorrelation or the non-independence of samples, no statistics were performed on these data but means and standard deviations are presented to demonstrate apparent differences in relative abundance among ecoregions.

\section{Results}

\subsection{Distribution}

Incidental and targeted surveys conducted by DFO captured River Darters in all three biogeographic ecoregions that were sampled; the Saskatchewan-Nelson, Southern Hudson Bay-James Bay, and Great Lakes-Upper St. Lawrence ecoregions (Tables 1 and 2). These included some large watersheds in the Saskatchewan-Nelson ecoregion such as the Rainy, Winnipeg, English, and Assiniboine rivers, and Lake Winnipeg, Dauphin Lake, and Lake Winnipegosis watersheds (Figure 1). Fewer collections were made in the Southern Hudson Bay-James Bay ecoregion (the Albany and Attiwapiskat river watersheds) and Great Lakes-Upper St. Lawrence ecoregion (Lake St. Clair watershed; Figure 1). Specimens collected by other researchers and catalogued in the Royal Ontario Museum from 1990-2014 were only sampled in the Saskatchewan-Nelson and Great Lakes-Upper St. Lawrence ecoregions (Table 3). 
River Darters were confirmed extant at all historical locations surveyed except in the Balne River. Although our sampling of historic sites was limited by road access (some earlier collections were made by flying into remote locations), we were successful in capturing River Darters in waterbodies where they were earlier found, and also in increasing the current known distribution in some watersheds (Figure 1). A notable collection since the 1990 review was the first record from Saskatchewan, single specimen collected in 1990 in the Saskatchewan River by R. Eakins (ROM60976) (Table 3).

\subsection{Relative Abundance}

A total of 1031 River Darters were captured during the incidental and targeted surveys (Tables 1 and 2) from 29 lakes and rivers. The majority of these fish were collected on the Assiniboine (402) and Rainy (342) rivers. Five-hundred and eighty-two (582) fish were collected as part of targeted surveys and the 2013 electrofishing survey in the Rainy River that were then used for relative abundance and biological assessments (Table 2). Relatively large collections ( $>20$ individuals) were made in the Rainy, English, Sturgeon, and Assiniboine rivers, and the Lake of the Woods, Lake Winnipeg, Lake St. Joseph, and Badesdawa Lake (Table 1). Relative abundance varied among sampling sites, with a number of sites in the Saskatchewan-Nelson and Southern Hudson Bay-James Bay ecoregions, including the Assiniboine, English and Rainy rivers, Lake Winnipeg, Lake St. Joseph, Badesdawa Lake, and Lake of the Woods, exhibiting relatively high ( $>50$ fish/ha) densities (Table 2). Most notably, low densities occurred in the historical southern Ontario sites (Thames and Sydenham rivers) in the Great Lakes-Upper St. Lawrence ecoregion, while higher mean densities were apparent in both the Southern Hudson Bay-James Bay and Saskatchewan-Nelson ecoregions (Figure 2). The mean density of River Darters was high in some locations (e.g., the lower English River, Lake St. Joseph, and the Assiniboine River) and low in others (Thames, Sydenham, and Balne rivers, and Red Lake; Figure 2).

\subsection{Life History Characteristics}

Mean length and weight varied among waterbodies and appeared influenced by sample date, as late summer and fall collections contained many small age-0 individuals (Table 4). The Assiniboine and Rainy Rivers contained the largest individuals and had the greatest age range of any of the populations (Table 4; Figure 3). Age-0 fish were prominent in all the late-summer/fall collections, making up the vast majority of individuals captured in many sites. Growth in the Saskatchewan-Nelson ecoregions appears relatively linear (Figure 3), with fish growing at approximately $10 \mathrm{~mm} /$ year. Growth in the Southern Hudson Bay-James Bay ecoregion appears to be slower than growth in the Saskatchewan-Nelson ecoregion for age-0 and age-1 fish (Figure 4); no fish older than age-1 were collected from the Southern Hudson Bay-James Bay ecoregion.

It was generally possible to identify the gender of fish age- 1 and older, suggesting that the River Darters in these collections mature after their first winter. Sex ratios were highly skewed towards female dominance (Table 4), with only the populations in the Sturgeon River and Badesdawa Lake demonstrating balanced sex ratios.

\subsection{Diet}

Stomach content assessment indicated that River Darters utilize a wide prey base, likely reflecting the dynamic habitat conditions (lotic vs. lentic, June vs. September/October) under which the sampling occurred. While each waterbody appeared to have its own somewhat unique prey base, in the June samples certain diet items were common among sites including chironomids (Chironomidae), caddisflies (Hydropsychidae, Polycentropodidae, Phryganeidae, and Leptoceridae) and mayflies (Ephemerellidae, Ephemeridae, Heptageniidae, Baetidae, and Leptophlebiidae; Figure 5). Chironomids, mayflies, and caddisflies were also present in fall diets, but other prey items, notably zooplankton and gastropods (Lymnaeidae), also became important diet items (Figure 5). 


\subsection{Habitat Characteristics}

River Darters were captured in a wide variety of habitats. This included multiple collections from both lotic and lentic waterbodies. In targeted, 2014 sampling efforts, River Darters were collected at a depth range from $2-5 \mathrm{~m}$, with water temperatures ranging $8.5-15.6{ }^{\circ} \mathrm{C}$, and turbidity ranging 0.4-6.4 NTUs (Table 5). Dissolved oxygen concentrations and $\mathrm{pH}$ levels at sampling time were well within the range of tolerance for a fish with a wide North American distribution (Table 5). Habitat data were also available for many of the incidental River Darter collections from the Assiniboine River, in depths ranging $0.28-3.0 \mathrm{~m}$, velocities $0.02-0.99 \mathrm{~m} / \mathrm{s}$, and temperatures $20.5-25.9^{\circ} \mathrm{C}$.
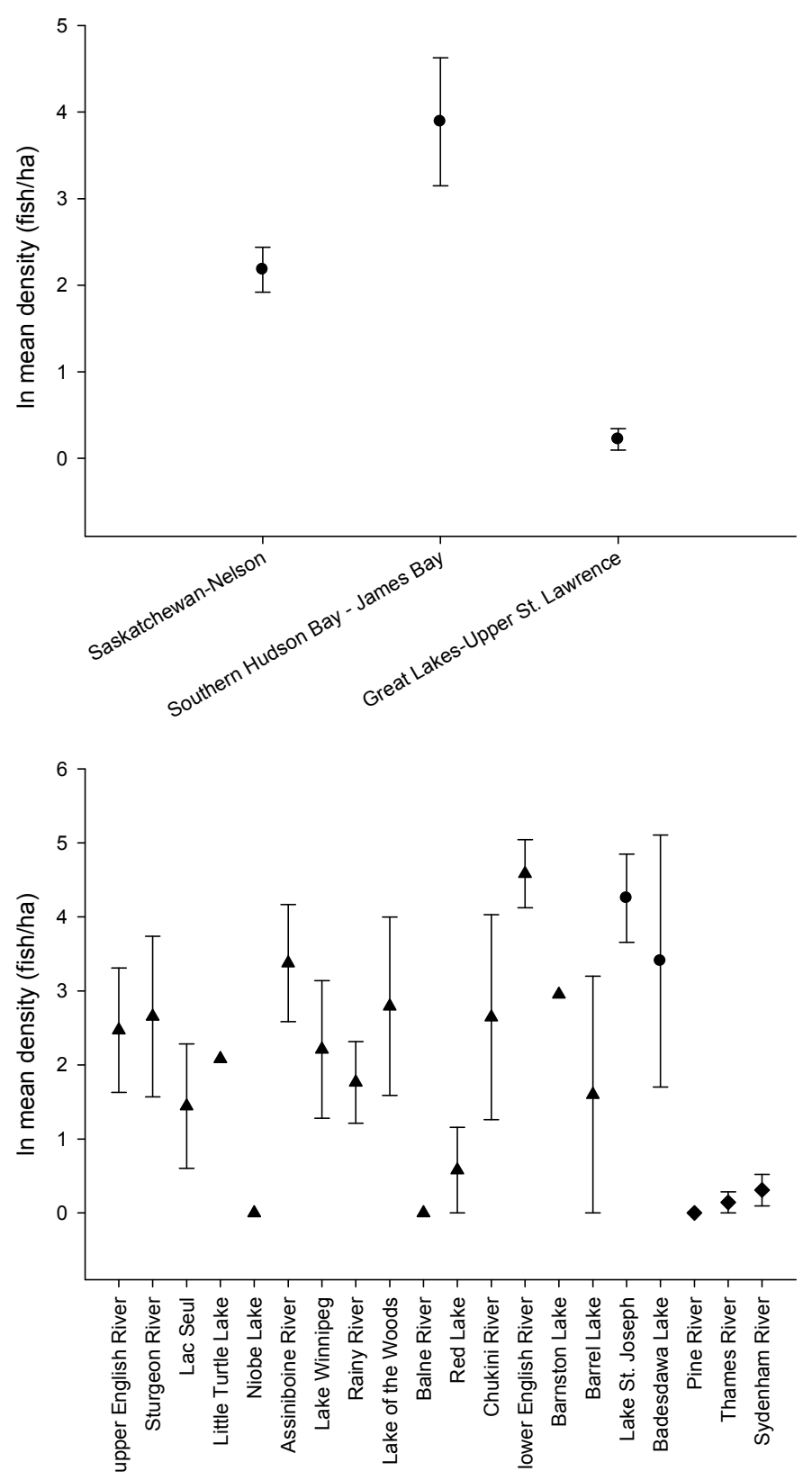

Figure 2. Mean River Darter density from three ecoregions (top panel) and individual sites (bottom panel) recently (2012-2014) sampled by DFO. The Saskatchewan-Nelson River ecoregion ( $\boldsymbol{\Delta}$ in lower panel) is represented by the fifteen 2014 sampling sites (64 trawls); Southern Hudson Bay-James Bay ecoregion ( $\bullet$ in lower panel) is represented by two sites (7 trawls); and Great Lakes-Upper St. Lawrence ecoregion ( $\downarrow$ in lower panel) is represented by three sites ( 8 trawls). Error bars represent \pm standard error. 

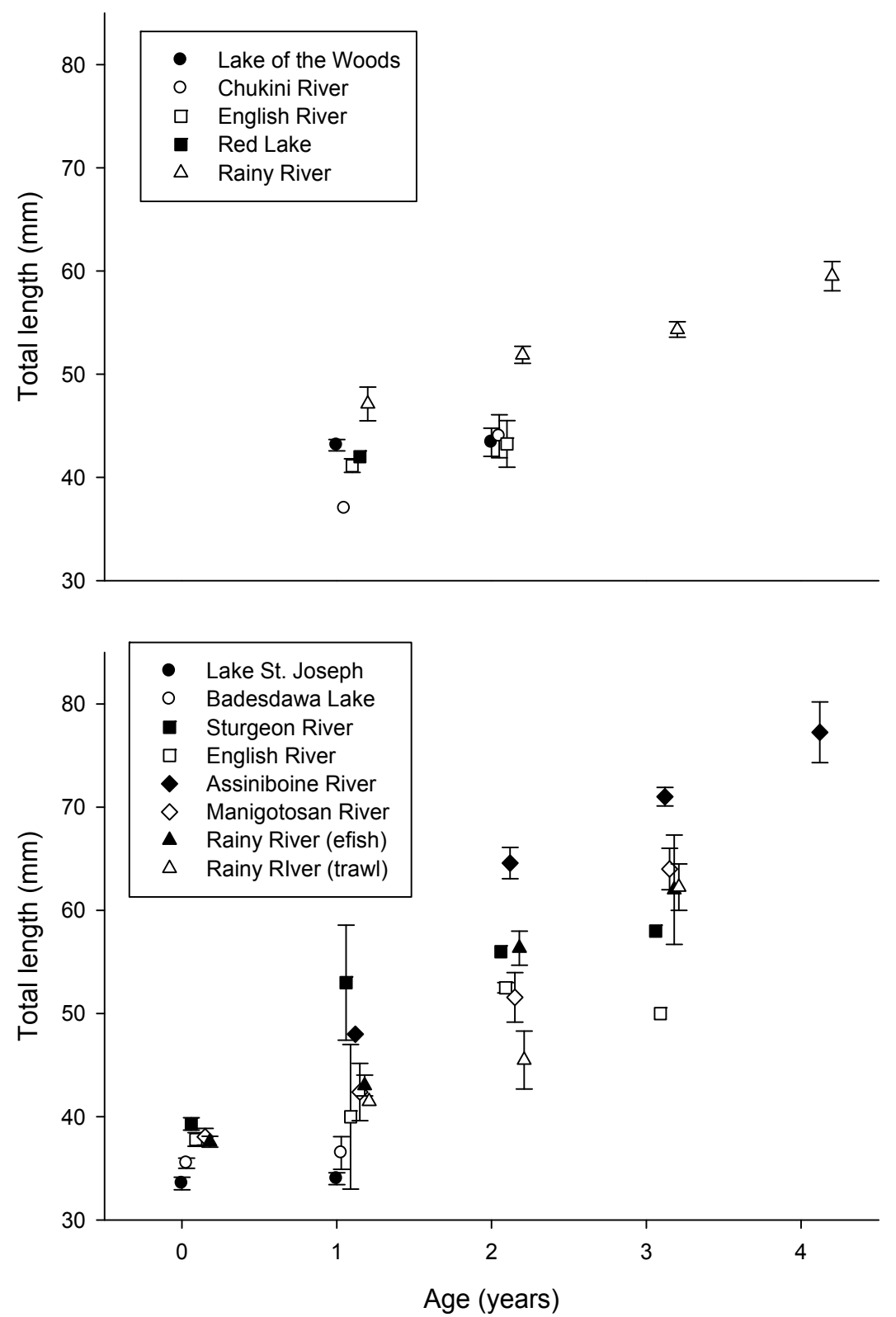

Figure 3. Mean length-at-age for River Darters collected from sites recently (2012-2014) sampled by DFO in the spring (top panel) and the fall (bottom panel). Error bars represent \pm standard error. 


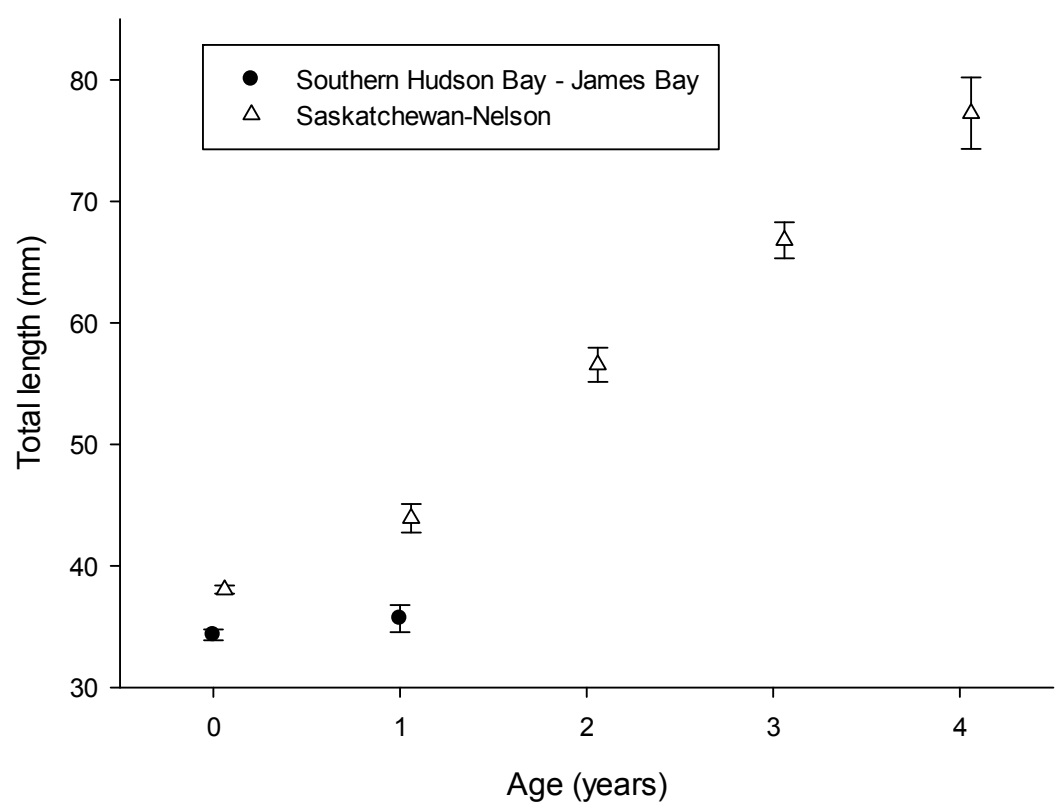

Figure 4. Mean length-at-age for River Darters sampled from two ecoregions, Southern Hudson Bay-James Bay and Saskatchewan-Nelson River, in the fall seasons of 2013-2014. Error bars represent \pm standard error.

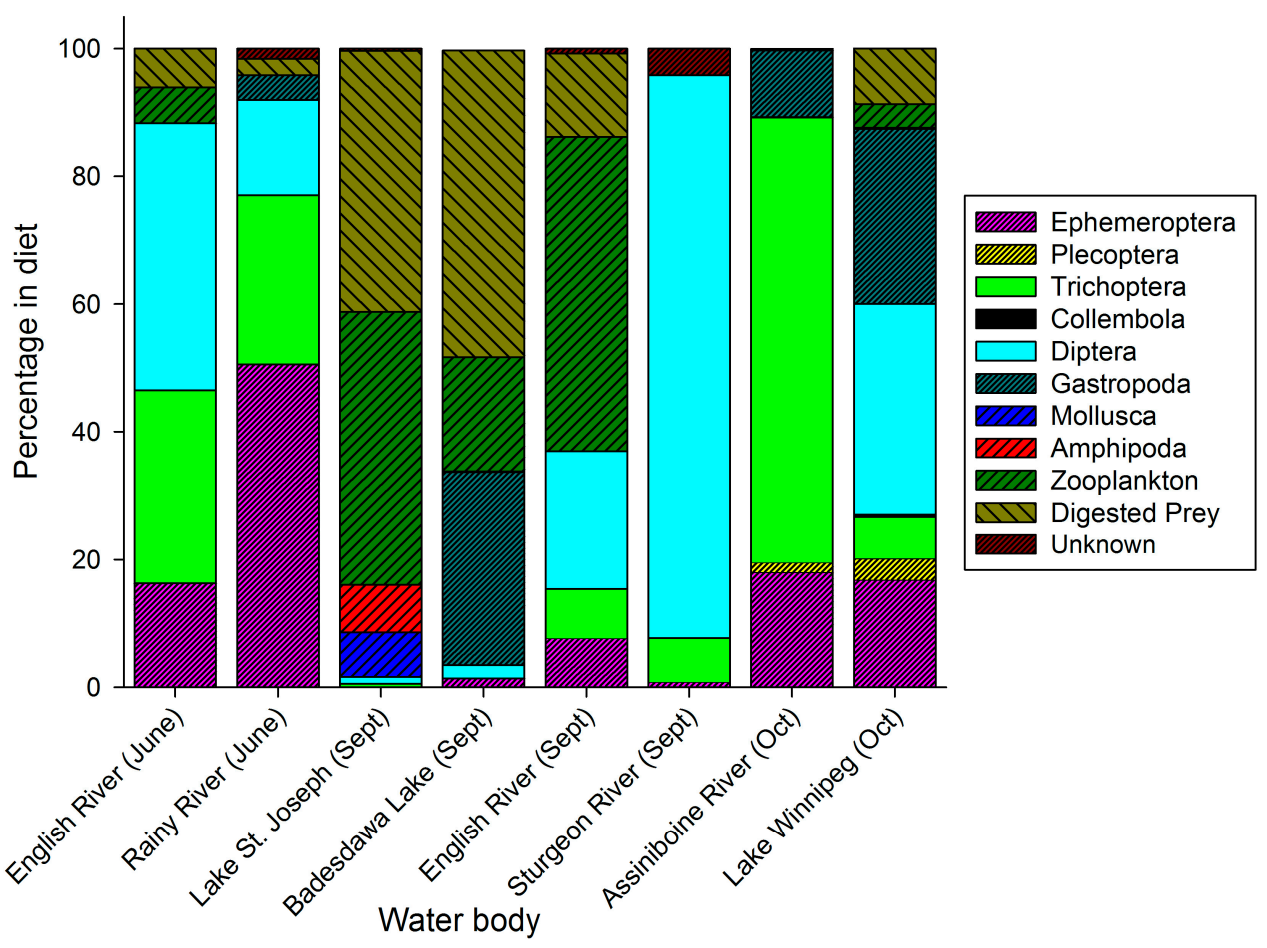

Figure 5. Common diet items in the stomach contents of seven River Darter populations collected in 2013-2014. 
Table 1. Sampling site and date, ecoregion, gear type, and the number of River Darters captured by DFO for surveys where River Darters were incidentally captured. Whether River Darters were previously found at that site is also indicated.

\begin{tabular}{|c|c|c|c|c|c|c|}
\hline Ecoregion & Waterbody & Latitude and Longitude & Historic Site & Date(s) & Gear Type & Number Captured \\
\hline Saskatchewan-Nelson & Assiniboine River & Captures from 99 sites & Yes & $28 / 08 / 1996$ to $23 / 07 / 2009$ & trawl and boat efishing & 366 \\
\hline Saskatchewan-Nelson & Bird Lake/Bird River & $50^{\circ} 28.984 ;-95^{\circ} 15.981$ & New & $14 / 08 / 2003$ to $02 / 10 / 2003$ & boat efishing & 19 \\
\hline Saskatchewan-Nelson & Boundary Creek & $50^{\circ} 30.521 ;-96^{\circ} 58.537$ & New & $23 / 06 / 2004$ & backpack efishing & 1 \\
\hline Saskatchewan-Nelson & Crowduck Lake & $50^{\circ} 06.389 ;-95^{\circ} 16.198$ & New & $01 / 09 / 2009$ and $02 / 09 / 2009$ & seine & 4 \\
\hline Saskatchewan-Nelson & Icelandic River & $50^{\circ} 57.887 ;-97^{\circ} 02.329$ & New & $16 / 07 / 2004$ & backpack efishing & 1 \\
\hline Saskatchewan-Nelson & La Salle River & $49^{\circ} 41.659 ;-97^{\circ} 15.748$ & New & $17 / 04 / 2002$ & backpack efishing & 1 \\
\hline Saskatchewan-Nelson & Lake Winnipeg & $51^{\circ} 47.315 ;-96^{\circ} 52.347$ & Yes & $27 / 07 / 2006$ & trawl & 1 \\
\hline Saskatchewan-Nelson & Manigotogan River & $51^{\circ} 06.091 ;-96^{\circ} 17.029$ & Yes & $03 / 09 / 2003$ & boat efishing & 9 \\
\hline Saskatchewan-Nelson & Norquay Channel & $49^{\circ} 31.921 ;-97^{\circ} 51.847$ & New & $28 / 05 / 2002$ & backpack efishing & 4 \\
\hline Saskatchewan-Nelson & Ochre River & $50^{\circ} 54.266 ;-99^{\circ} 49.150$ & New & 10/05/1999 & unknown & 1 \\
\hline Saskatchewan-Nelson & Ochre River & $51^{\circ} 03.083 ;-99^{\circ} 47.192$ & New & $23 / 07 / 2002$ & backpack efishing & 4 \\
\hline Saskatchewan-Nelson & Rainy River & $48^{\circ} 38.677 ;-94^{\circ} 05.680$ & Yes & $28 / 07 / 2004$ & boat efishing & 2 \\
\hline Saskatchewan-Nelson & Riviere Aux Marais & $49^{\circ} 08.004 ;-97^{\circ} 17.523$ & New & $23 / 07 / 2003$ & backpack efishing & 1 \\
\hline Saskatchewan-Nelson & Seine River Diversion & $49^{\circ} 41.840 ;-97^{\circ} 05.985$ & New & $30 / 07 / 2004$ & backpack efishing & 18 \\
\hline Saskatchewan-Nelson & Swan River & $52^{\circ} 13.668 ;-100^{\circ} 59.552$ & New & $05 / 08 / 2004$ & backpack efishing & 10 \\
\hline Saskatchewan-Nelson & Unnamed tributary to Boyne River & $49^{\circ} 32.464 ;-98^{\circ} 24.885$ & New & $27 / 08 / 2002$ & backpack efishing & 1 \\
\hline Saskatchewan-Nelson & Wilson River & $51^{\circ} 11.973 ;-100^{\circ} 06.277$ & New & $30 / 06 / 2004$ & backpack efishing & 1 \\
\hline Saskatchewan-Nelson & Winnipeg River & $50^{\circ} 13.373 ;-95^{\circ} 34.397$ & Yes & $15 / 09 / 2003$ and $16 / 09 / 2003$ & boat efishing & 2 \\
\hline Saskatchewan-Nelson & Vermillion River & $51^{\circ} 10.185 ;-100^{\circ} 03.225$ & New & $20 / 07 / 2004$ & backpack efishing & 3 \\
\hline
\end{tabular}


Table 2. Sampling site and date, ecoregion, gear type, effort (number of trawls or electrofishing [efishing] transects), the number of individuals captured, and mean density ( \pm standard error) from recent DFO-led targeted River Darter surveys. Whether River Darters were previously found at that site is also indicated.

\begin{tabular}{|c|c|c|c|c|c|c|c|c|c|}
\hline Ecoregion & Waterbody & $\begin{array}{l}\text { Latitude and } \\
\text { Longitude }\end{array}$ & $\begin{array}{l}\text { Historic } \\
\text { Site }\end{array}$ & Date(s) & Gear Type & Effort & $\begin{array}{l}\text { Mean Length } \\
\text { (m) }\end{array}$ & $\begin{array}{l}\text { River Darters } \\
\text { Captured }\end{array}$ & $\begin{array}{l}\text { Density } \\
\text { (Fish/ha) }\end{array}$ \\
\hline \multirow{4}{*}{ Saskatchewan-Nelson } & \multirow{4}{*}{ Rainy River } & $48^{\circ} 34.155 ;-93^{\circ} 27.610$ & \multirow{4}{*}{ Yes } & $19 / 06 / 2013$ to $22 / 06 / 2013$ & trawl & 60 & 306.0 & 152 & $52.0(11.3)$ \\
\hline & & $48^{\circ} 30.926 ;-93^{\circ} 40.764$ & & $08 / 08 / 2013$ to $13 / 08 / 2013$ & boat efishing & 24 & 1609.3 & 168 & $17.4(7.2)$ \\
\hline & & $48^{\circ} 32.474 ;-93^{\circ} 30.261$ & & $11 / 09 / 2013$ to $12 / 09 / 2013$ & trawl & 12 & 282.0 & 17 & $20.7(8.6)$ \\
\hline & & $48^{\circ} 31.687 ;-93^{\circ} 35.274$ & & $13 / 06 / 2014$ & trawl & 2 & 365.0 & 3 & $10.3(10.3)$ \\
\hline Saskatchewan-Nelson & Lake of the Woods & $48^{\circ} 58.276 ;-94^{\circ} 33.213$ & Yes & $13 / 06 / 2014$ & trawl & 5 & 174.8 & 21 & $97.5(65.6)$ \\
\hline Saskatchewan-Nelson & Balne River & $49^{\circ} 48.933 ;-94^{\circ} 13.300$ & Yes & $14 / 06 / 2014$ & trawl & 4 & 151.8 & 0 & - \\
\hline Saskatchewan-Nelson & Red Lake & $51^{\circ} 05.279 ;-93^{\circ} 48.536$ & Yes & $15 / 06 / 2014$ & trawl & 7 & 121.0 & 1 & $8.1(8.1)$ \\
\hline Saskatchewan-Nelson & Chukini River & $50^{\circ} 56.117 ;-93^{\circ} 36.264$ & New & $16 / 06 / 2014$ & trawl & 3 & 173.3 & 5 & $43.9(32.2)$ \\
\hline \multirow{2}{*}{ Saskatchewan-Nelson } & English River & $50^{\circ} 37.531 ;-93^{\circ} 15.808$ & \multirow{2}{*}{ Yes } & $16 / 06 / 2014$ & trawl & 5 & 134.2 & 27 & $140.4(53.5)$ \\
\hline & (lower and upper) & $49^{\circ} 38.028 ;-91^{\circ} 21.913$ & & $13 / 09 / 2014$ & trawl & 6 & 273.3 & 17 & $37.6(20.4)$ \\
\hline Saskatchewan-Nelson & Barnston Lake & $50^{\circ} 34.567 ;-93^{\circ} 28.223$ & Yes & $16 / 06 / 2014$ & trawl & 1 & 220.0 & 1 & 18.8 \\
\hline Saskatchewan-Nelson & Barrel Lake & $49^{\circ} 39.468 ;-91^{\circ} 29.369$ & Yes & $19 / 06 / 2014$ & trawl & 2 & 215.0 & 1 & $11.8(11.8)$ \\
\hline Saskatchewan-Nelson & Sturgeon River & $50^{\circ} 06.886 ;-91^{\circ} 43.192$ & New & $14 / 09 / 2014$ & trawl & 5 & 411.2 & 26 & $49.5(20.2)$ \\
\hline Saskatchewan-Nelson & Lac Seul & $50^{\circ} 12.163 ;-91^{\circ} 50.705$ & Yes & $14 / 09 / 2014$ & trawl & 4 & 330.0 & 2 & $8.8(5.4)$ \\
\hline Saskatchewan-Nelson & Little Turtle Lake & $48^{\circ} 46.804 ;-92^{\circ} 36.968$ & $\mathrm{New}$ & $16 / 09 / 2014$ & trawl & 1 & 570.0 & 1 & 7.0 \\
\hline Saskatchewan-Nelson & Niobe Lake & $48^{\circ} 43.631 ;-91^{\circ} 19.549$ & $\mathrm{~N} / \mathrm{A}$ & $16 / 09 / 2014$ & trawl & 1 & 380.0 & 0 & - \\
\hline Saskatchewan-Nelson & Assiniboine River & $49^{\circ} 42.245 ;-99^{\circ} 40.109$ & Yes & $09 / 10 / 2014$ & trawl & 8 & 169.9 & 36 & $96.8(40.4)$ \\
\hline Saskatchewan-Nelson & Lake Winnipeg & $51^{\circ} 07.195 ;-96^{\circ} 21.271$ & Yes & $08 / 10 / 2014$ & trawl & 10 & 66.0 & 33 & $182.1(12.5)$ \\
\hline Southern Hudson Bay-James Bay & Lake St. Joseph & $51^{\circ} 05.877 ;-90^{\circ} 17.529$ & Yes & $12 / 09 / 2014$ & trawl & 4 & 305.0 & 30 & $117.8(68.5)$ \\
\hline Southern Hudson Bay-James Bay & Badesdawa Lake & $51^{\circ} 43.728 ;-89^{\circ} 46.994$ & New & $12 / 09 / 2014$ & trawl & 3 & 306.7 & 38 & $109.7(55.5)$ \\
\hline Great Lakes-Upper St. Lawrence & Pine River & $48^{\circ} 02.615 ;-89^{\circ} 30.579$ & $\mathrm{~N} / \mathrm{A}$ & $17 / 09 / 2014$ & trawl & 1 & 730.0 & 0 & - \\
\hline Great Lakes-Upper St. Lawrence & Thames River & $42^{\circ} 36.586 ;-81^{\circ} 49.170$ & Yes & $24 / 06 / 2014$ to $26 / 06 / 2014$ & trawl & 26 & 100.0 & 1 & $1.5(1.5)$ \\
\hline Great Lakes-Upper St. Lawrence & Sydenham River & $42^{\circ} 35.887 ;-82^{\circ} 21.545$ & Yes & $20 / 09 / 2012$ & trawl & 24 & 100.0 & 2 & $3.3(2.3)$ \\
\hline
\end{tabular}


Table 3. ROM River Darter collection records from 1990-2014.

\begin{tabular}{cccc}
\hline Waterbody & Date & Latitude and Longitude & Catalogue Number \\
\hline Saskatchewan River & $24 / 08 / 1990$ & $53^{\circ} 40.800 ;-103^{\circ} 23.700$ & 60976 \\
Thames River & $26 / 07 / 1991$ & $42^{\circ} 35.467 ;-81^{\circ} 53.066$ & 62116 \\
Barnston Lake & $18 / 08 / 1993$ & $50^{\circ} 34.083 ;-93^{\circ} 30.300$ & 68403 \\
Barnston Lake & $18 / 08 / 1993$ & $50^{\circ} 34.133 ;-93^{\circ} 30.767$ & 68407 \\
Barnston Lake & $18 / 08 / 1993$ & $50^{\circ} 34.100 ;-93^{\circ} 30.466$ & 68523 \\
Barnston Lake & $19 / 08 / 1993$ & $50^{\circ} 35.250 ;-93^{\circ} 30.400$ & 68524 \\
Barnston Lake & $19 / 08 / 1993$ & $50^{\circ} 34.333 ;-93^{\circ} 31.400$ & 68529 \\
English River & $23 / 07 / 1994$ & $50^{\circ} 34.930 ;-93^{\circ} 27.719$ & 87830 \\
Bear Creek & $05 / 08 / 1997$ & $42^{\circ} 47.266 ;-82^{\circ} 18.533$ & 70777 \\
Bear Creek & $07 / 08 / 1997$ & $42^{\circ} 45.933 ;-82^{\circ} 19.766$ & 70779 \\
Bear Creek & $07 / 08 / 1997$ & $42^{\circ} 45.933 ;-82^{\circ} 19.766$ & CID $96376 \mathrm{~B}$ \\
Bear Creek & $07 / 08 / 1997$ & $42^{\circ} 45.933 ;-82^{\circ} 19.766$ & CID 96830 W \\
Sydenham River (East) & $07 / 08 / 1997$ & $42^{\circ} 35.417 ;-82^{\circ} 16.033$ & CID $96972 \mathrm{~W}$ \\
Sydenham River (East) & $01 / 10 / 1997$ & $42^{\circ} 35.850 ;-82^{\circ} 22.066$ & CID $105941 \mathrm{~W}$ \\
Sturgeon River & $08 / 08 / 1998$ & $48^{\circ} 39.266 ;-94^{\circ} 01.550$ & CID $106175 \mathrm{E}$ \\
Sydenham River & $18 / 06 / 2001$ & $42^{\circ} 35.300 ;-82^{\circ} 13.250$ & 72613 \\
Rainy River & $19 / 09 / 2002$ & $48^{\circ} 35.939 ;-93^{\circ} 25.892$ & 75863 \\
Rainy River & $19 / 09 / 2002$ & $48^{\circ} 36.338 ;-93^{\circ} 25.111$ & 75867 \\
Rainy River & $19 / 09 / 2002$ & $48^{\circ} 36.445 ;-93^{\circ} 24.831$ & 75869 \\
North Sydenham River & $10 / 09 / 2003$ & $42^{\circ} 39.442 ;-82^{\circ} 22.540$ & 79743 \\
North Sydenham River & $11 / 09 / 2003$ & $42^{\circ} 38.927 ;-82^{\circ} 22.414$ & CID $120653 \mathrm{E}$ \\
Lake St Clair & $10 / 08 / 2006$ & $42^{\circ} 19.762 ;-82^{\circ} 26.767$ & 89210 \\
Lake St Clair & $16 / 07 / 2013$ & $42^{\circ} 20.816 ;-82^{\circ} 25.650$ & 95703 \\
\hline
\end{tabular}


Table 4. Mean total length $\left(l_{t}\right)$ length range $(\mathrm{mm})$, mean weight $(\mathrm{g})$, and sex ratio for River Darters captured and assessed from DFO-led surveys. Numbers in brackets represent \pm standard error. The Royal Ontario Museum (ROM) catalogue number is provided when available.

\begin{tabular}{|c|c|c|c|c|c|c|c|c|c|c|}
\hline Waterbody & Date(s) & $\begin{array}{l}\text { ROM Catalogue } \\
\text { Number }\end{array}$ & $\begin{array}{l}\text { Sample Size for } \\
L_{t} \text { and Weight }\end{array}$ & $\begin{array}{c}\text { Mean } L_{t} \\
(\mathrm{~mm})\end{array}$ & $\begin{array}{c}\text { Length } \\
\text { Range (mm) }\end{array}$ & $\begin{array}{c}\text { Mean } \\
\text { Weight (g) }\end{array}$ & Sex Ratio & $\begin{array}{c}\text { Sample Size } \\
\text { for Aging }\end{array}$ & $\begin{array}{c}\text { Mean Age } \\
\text { (Years) }\end{array}$ & Age Range \\
\hline \multirow{3}{*}{ Rainy River } & $19 / 06 / 2013$ to $22 / 06 / 2013$ & & $145(1) ; 144(w)$ & $53.0(0.4)$ & $43-67.5$ & $1.2(0.04)$ & $60^{x}: 719$ & 98 & 2.6 & $1-4$ \\
\hline & $08 / 08 / 2013$ to $13 / 08 / 2013$ & & 167 & $42.5(0.7)$ & $30-72$ & $0.6(0.04)$ & & 65 & 0.7 & $0-3$ \\
\hline & $11 / 09 / 2013$ to $12 / 09 / 2013$ & & 16 & $46.7(1.9)$ & $40-64.5$ & $0.8(0.12)$ & $30^{\top}: 13 q$ & 9 & 2.1 & $1-3$ \\
\hline Lake of the Woods & $13 / 06 / 2014$ & 096714 & 17 & $43.6(0.5)$ & $40-47$ & $0.6(0.02)$ & $40^{\top}: 119$ & 13 & 1.4 & $1-2$ \\
\hline Red Lake & $15 / 06 / 2014$ & & 1 & 42 & & 0.43 & $00^{\top}: 19$ & 1 & 1 & \\
\hline Chukini River & $16 / 06 / 2014$ & 096711 & 5 & $42.4(1.8)$ & $37-48$ & $0.6(0.07)$ & $10^{\star}: 19$ & 4 & 1.8 & $1-2$ \\
\hline \multirow{2}{*}{ English River } & $16 / 06 / 2014$ & & 27 & $41.4(0.6)$ & $35-47$ & $0.5(0.03)$ & $70^{\top}: 17 \%$ & 25 & 1.2 & $1-2$ \\
\hline & $13 / 09 / 2014$ & 096708 & 17 & $40.3(1.6)$ & $30-53$ & $0.5(0.06)$ & $2 \sigma^{\gamma}: 14$ 우 & 15 & 0.6 & $0-3$ \\
\hline Barnston Lake & $16 / 06 / 2014$ & 096715 & 1 & 41 & & 0.38 & & & & \\
\hline Barrel Lake & $19 / 06 / 2014$ & 096717 & 1 & 48.5 & & 0.5 & & & & \\
\hline Sturgeon River & $14 / 09 / 2014$ & 096713 & 26 & $42.3(1.4)$ & $35-60$ & $0.7(0.09)$ & $130^{\top}: 119$ & 21 & 0.4 & $0-3$ \\
\hline Lac Seul & $14 / 09 / 2014$ & 096712 & 1 & 30 & & 0.2 & & & & \\
\hline Little Turtle Lake & $16 / 09 / 2014$ & 096707 & 1 & 41 & & 0.5 & & & & \\
\hline Assiniboine River & $09 / 10 / 2014$ & 096716 & 36 & $69.3(1.4)$ & $48-93$ & $2.9(0.20)$ & $00^{\top}: 359$ & 36 & 2.8 & $1-4$ \\
\hline Lake Winnipeg & 08/10/2014 & & 31 & $43.5(1.6)$ & $33-66$ & $0.7(0.10)$ & $80^{\top}: 18 ?$ & 31 & 0.8 & $0-3$ \\
\hline Lake St. Joseph & $12 / 09 / 2014$ & 096709 & 30 & $33.9(0.6)$ & $30-40$ & $0.3(0.02)$ & $80^{r}: 17 \%$ & 27 & 0.1 & $0-1$ \\
\hline Badesdawa Lake & $12 / 09 / 2014$ & 096710 & 32 & $36.1(0.4)$ & $32-43$ & $0.3(0.01)$ & $110^{\Uparrow}: 149$ & 22 & 0.3 & $0-1$ \\
\hline
\end{tabular}

Table 5. Mean measured habitat parameters, including depth, temperature, $\mathrm{pH}$, turbidity, and dissolved oxygen from selected River Darter collection sites.

\begin{tabular}{|c|c|c|c|c|c|c|}
\hline Waterbody & Date(s) & Depth (m) & Temperature $\left({ }^{\circ} \mathrm{C}\right)$ & $\mathrm{pH}$ & Turbidity (NTUs) & Dissolved Oxygen $(\mathrm{mg} / \mathrm{L})$ \\
\hline Lake of the Woods & $13 / 06 / 2014$ & 3.7 & 15.63 & 7.55 & 6.3 & 9.12 \\
\hline Red Lake & $15 / 06 / 2014$ & 3.4 & 8.52 & 7.51 & 0.4 & 10.39 \\
\hline Chukini River & $16 / 06 / 2014$ & 3.6 & 10.78 & 7.56 & 2.5 & 9.59 \\
\hline & $16 / 06 / 2014$ & 3.5 & 12.65 & 7.71 & 5.6 & 10.16 \\
\hline English River & $13 / 09 / 2014$ & 4.6 & 14.07 & 8.10 & 1.2 & 9.63 \\
\hline Barnston Lake & $16 / 06 / 2014$ & 3.0 & 13.79 & 7.56 & 5.8 & 10.03 \\
\hline Barrel Lake & $19 / 06 / 2014$ & 3.8 & 15.41 & 7.62 & 1.3 & 9.02 \\
\hline Lake St. Joseph & $12 / 09 / 2014$ & 4.8 & 14.44 & 7.10 & 1.3 & 9.67 \\
\hline Badesdawa Lake & $12 / 09 / 2014$ & 2.5 & 10.45 & 7.91 & 3.1 & 10.54 \\
\hline Sturgeon River & $14 / 09 / 2014$ & 5.0 & 14.56 & 8.02 & 1.1 & 10.01 \\
\hline Lac Seul & $14 / 09 / 2014$ & 4.2 & 13.32 & 7.89 & 1.7 & 9.17 \\
\hline Little Turtle Lake & $16 / 09 / 2014$ & 2.0 & 12.96 & 7.80 & 5.8 & 9.90 \\
\hline
\end{tabular}




\section{Discussion}

These collections demonstrate that the River Darter continues to be broadly distributed throughout its historic range in Canada, and are present in more sites and at a higher abundance than was previously believed. We believe that these improvements are likely due to the use of more appropriate sampling techniques (e.g., mini-Missouri trawls) than any actual expansion in the species range or large increases in population sizes. A similar expansion of the known distribution of River Darters occurred in Pennsylvania when trawls were employed in areas that were not previously sampled (e.g., using trawls in the upper portion of the Ohio River) [4]. The most northern historic Canadian sites are not road accessible, so it was not possible to re-sample those areas given the limited budget of this project. Therefore, the current status of the River Darter in those remote sites is not known.

Relatively robust River Darter populations were apparent in a number of lakes and rivers. An earlier review suggested that River Darters were not abundant in Canada [6], with the largest collection at that time being 10 specimens. However, the River Darter is locally abundant in large, turbid rivers in the United States [1,13]. Our surveys resulted in many collections with more than 10 specimens, indicating that River Darters are likely more common in Canadian waters than originally believed. The higher catches are likely a result of using a type of gear (trawling) that was more effective at sampling benthic fish communities in deeper water than seines or electrofishing. While other survey techniques (seining, electrofishing) have captured River Darters, it is apparent that the habitats exploited by most River Darters are best sampled using trawls. The mini-Missouri trawl used in this study has been used to sample many rare benthic fishes including sturgeons, darters, and madtoms [14,15], emphasizing the importance of using the best sampling technique for the habitats and fishes being sampled.

Our large collections in the core of the species Canadian range (southern Manitoba and northwestern Ontario) suggest that River Darter populations remain healthy in at least two of the ecoregions (Saskatchewan-Nelson and Southern Hudson Bay-James Bay) in their Canadian Range. This is not the case for the Great Lakes-Upper St. Lawrence ecoregion, where very few individuals have been collected despite significant trawling effort in rivers where historic River Darter records exist. Populations in the Great Lakes-Upper St. Lawrence ecoregion face different and likely more severe threats than populations in the other ecoregions, where development pressures are less severe [9]. Specific threats in the Great Lakes-Upper St. Lawrence ecoregion include invasive species such as the Round Goby (Neogobius melanostomus), whose distribution and habitat overlaps with River Darter in the Great Lakes-Upper St. Lawrence ecoregion and may negatively impact the River Darter through direct competition for food resources [16], and increased levels of industrial and agricultural effluents, nutrients, sedimentation, all of which will degrade River Darter habitat [9]. There are different threats in the other ecoregions, particularly dams and impoundments in the Saskatchewan-Nelson ecoregion which reduce stream gradients and increase fine substrates, conditions which have previously been linked to declines in River Darter populations $[17,18]$. While we do not know whether it is underlying differences in geology resulting in different habitat characteristics, climactic regimes, or anthropogenic threats (or a combination of all three), our data clearly demonstrate reduced River Darter populations in the Great Lakes-Upper St. Lawrence ecoregion.

Prior to this assessment, the biology of the River Darter in Canadian populations was poorly known. In Illinois, River Darters mature as early as age-1 and reach a maximum age-3 [17] or age-4 [19]. The same age-at-maturity (1) and maximum age (4) were apparent in the populations assessed in this study, indicating that these life history traits are common across the species' range studied to date. River Darters in Manitoba and northwestern Ontario grow slowly, adding approximately $10 \mathrm{~mm}$ /year in length. No other estimates of annual growth were available in the literature. River Darters also generally appear to have a skewed sex ratio, with a predominance of females in most of the assessed populations. This phenomenon is found in many fishes, and there are a number of explanations for differential survival of the sexes including increased mortality due to predation, differences in growth rate and an associated survival trade-off, or differences in reproductive activity or maturation 
schedule [20]. The high female dominance may indicate poor recent recruitment events for these River Darter populations if males have lower survival and females become dominant at older ages. Alternatively, females may simply be more vulnerable to the gear (trawls, electrofishing) used in this study, although we are unable to posit why this might occur.

Diet patterns observed in this assessment were consistent with the River Darter diet literature. River Darters feed primarily during daylight hours and consume a wide variety of food items that changes seasonally $[17,21]$. In Illinois and Manitoba, stomach contents included Diptera, Trichoptera, Ephemeroptera, Crustacae, and fish eggs [17,21]. A similar wide breadth of diet and seasonal influences were observed in this assessment. In addition to Diptera, Trichoptera, and Ephemeroptera, which were dominant spring prey items, zooplankton became an important diet item in fall-sampled sites. This seasonal shift in diet was apparent in our only location with fish collected in both June and September, the English River, where zooplankton became an increasingly important fall prey item. This shift might reflect prey availability, or the high proportion of smaller, age-0 River Darters present in the fall samples. In Alabama and Tennessee, snails can be an important component of the River Darter diet, varying seasonally with availability [22,23]. Snails were also were consumed by River Darters collected in September in Manitoba [21], and were also important prey items in the fall at some sites in this study.

Unlike many darter species that have specialized habitat niches, River Darters appear to be more broadly tolerant of a variety of habitat conditions. The findings of our targeted surveys concurred with the knowledge that River Darter populations occur in both rivers and lakes. Through much of its range, the River Darter is associated with a variety of substrates, moderate currents, and deeper water $[3,17,24-26]$. In our targeted surveys, River Darters were collected most frequently in moderate water velocities in rivers and at a wide range of depths in both rivers and lakes. River Darters are tolerant of turbid waters [21,26-28] and are a common, if not the most common, darter in turbid rivers [24,27]. Similarly, River Darters were collected over a wide range of turbidities in this survey, including large numbers in the turbid Assiniboine River. These more generalist habitat tendencies are most likely why the River Darter has one of the largest latitudinal distributions of all the darter species.

\section{Conclusions}

This study provides an important update on the distribution of the River Darter in its Canadian range. It provides the first relative density estimates available for the species, and confirms that River Darters in Canadian waters have similar life history traits and select comparable prey to conspecific populations located in the United States. It is clear that the River Darter populations in two of the ecoregions with the fewest anthropogenic stressors, Saskatchewan-Nelson and Southern Hudson Bay-James Bay, are widespread and relatively abundant. Conversely, populations in the highly populated Great Lakes-Upper St. Lawrence ecoregion are likely imperilled. Partly as a result of the data collected herein, the Committee on the Status of Endangered Wildlife in Canada, the scientific body that advises the Government of Canada on the conservation status of Canadian wildlife at risk, identified River Darters in the Great Lakes-Upper St. Lawrence ecoregion as endangered at their latest meeting in April 2016. The Saskatchewan-Nelson and Southern Hudson Bay-James Bay ecoregions were assessed as not at risk. Our work provides further support for the need to prioritize fish conservation activities in the southern Ontario portion of Great Lakes-Upper St. Lawrence ecoregion, an area which possesses many of the fishes of conservation concern in Canada and which is under increasing anthropogenic stress [29].

Acknowledgments: We thank David Rainho, Evan Timusk, Rick Elsner, and Robin Gaspardy for field sampling in northwestern Ontario, and Adam Batty, Bob Hrabik, Claire Herbert, Dave Herzog, Dave Ostendorf, Doug Leroux, Elliot Macdonald, Ernie Watson, Jeff Eastman, Jason Barnucz, Jeff Zeiller, Konrad Schmidt, Landan Fraser, Mark Lowdon, Mathew Martens, Maureen Forester, Patrick Nelson, Richard Penner, Stephanie Backhouse, Thomas Sheldon, and William Franzin for field sampling in Manitoba. Katelyn Kotska, Cheryl Widdifield, and Janice McKee completed specimen processing and aging. We are also grateful to Dave Milani for providing 
Manitoba data on River Dater distribution, and Erling Holm for confirming Royal Ontario Museum collection records. Jennie Pearce helped prepare the manuscript, and provided some valuable comments.

Author Contributions: All authors conceived the project, William M. Gardner and Douglas A. Watkinson conducted the field work, Thomas C. Pratt analysed the data, and Lynn D. Bouvier along with the other authors contributed to manuscript preparation.

Conflicts of Interest: The authors declare no conflict of interest.

\section{References}

1. Page, L.M.; Burr, B.M. Peterson Field Guide to Freshwater Fishes of North America North of Mexico, 2nd ed.; Houghton Mifflin Company: Boston, MD, USA, 2011; pp. 522-523.

2. Jelks, H.L.; Walsh, S.J.; Burkhead, N.M.; Contreras-Balderas, S.; Díaz-Pardo, E.; Hendrickson, D.A.; Lyons, J.; Mandrak, N.E.; McCormick, F.; Nelson, J.S.; et al. Conservation status of imperiled North American freshwater and diadromous fishes. Fisheries 2008, 33, 372-407. [CrossRef]

3. Scott, W.B.; Crossman, E.J. Freshwater fishes of Canada; Fisheries Research Board of Canada Bulletin 184: Ottawa, ON, Canada, 1973; pp. 806-808.

4. Freedman, J.A.; Stecko, T.D.; Criswell, R.W.; Stauffer, J.R., Jr. Extensions of the known ranges of Percina shumardi Girard and three species of Etheostoma (subgenus Nothonotus) in Pennsylvania. J. Pa. Acad. Sci. 2009, 83, 42-44.

5. Nature Serve Explorer: An Online Encyclopedia of Life. Version 7.1. Available online: http://www. natureserve.org (accessed on 26 August 2016).

6. Dalton, K.W. Status of the river darter, Percina shumardi, in Canada. Can. Field Nat. 1990, 104, 59-63.

7. Committee on the Status of Endangered Wildlife in Canada (COSEWIC). Guidelines for Recognizing Designatable Units. Available online: http://www.cosewic.gc.ca/images/Fig2FreshwaterBiogeographicZones_Eng.jpg (accessed on 25 November 2015).

8. Brown, J.H.; Lomolino, M.V. Biogeography, 2nd ed.; Sinauer Associates, Incorporated: Sunderland, MA, USA, 1998.

9. Committee on the Status of Endangered Wildlife in Canada (COSEWIC). COSEWIC Assessment and Status Report on the River Darter (Percina shumardi) in Canada; Committee on the Status of Endangered Wildlife in Canada: Ottawa, ON, Canada, 2016.

10. Herzog, D.P.; Barko, V.A.; Scheibe, J.S.; Hrabik, R.A.; Ostendorf, D.E. Efficacy of a benthic trawl for sampling small-bodied fishes in large river systems. N. Am. J. Fish. Manag. 2005, 25, 594-603. [CrossRef]

11. Herzog, D.P.; Ostendorf, D.E.; Hrabik, R.A.; Barko, V.A. The mini-Missouri trawl: A useful methodology for sampling small-bodied fishes in small and large river systems. J. Freshw. Ecol. 2009, 24, 103-108. [CrossRef]

12. International Council for the Exploration of the Sea (ICES). Workshop on age reading of European and American eel (WKAREA). In Proceedings of the ICES CM 2009/ACOM 48, Bordeaux, France, 20-24 April 2009.

13. Warren, L.W., Jr.; Burr, B.M.; Walsh, S.J.; Bart, H.L., Jr.; Cashner, R.C.; Etnier, D.A.; Freeman, B.J.; Kuhajda, B.R.; Mayden, R.L.; Robison, H.W.; et al. Diversity, distribution, and conservation status of the native freshwater fishes of the southern United States. Fisheries 2000, 25, 7-29. [CrossRef]

14. Phelps, Q.E.; Tripp, S.J.; Garvey, J.E.; Herzog, D.P.; Ostendorf, D.E.; Ridings, J.W.; Crites, J.W.; Hrabik, R.A. Habitat use during early life history infers recovery needs for shovelnose sturgeon and pallid sturgeon in the Middle Mississippi River. Trans. Am. Fish. Soc. 2010, 139, 1060-1068. [CrossRef]

15. Ridings, J.W.; Schell, S.R. Evaluation of a substrate-disturbing brail added to the mini-Missouri trawl for capture of madtoms, sculpins, and darters. J. Freshw. Ecol. 2014, 25, 337-344. [CrossRef]

16. Burkett, E.M.; Jude, D.J. Long-term impacts of invasive round goby Neogobius melanostomus on fish community diversity and diets in the St. Clair River, Michigan. J. Gt. Lakes Res. 2015, 41, 862-872. [CrossRef]

17. Thomas, D.L. An ecological study of four darters of the Genus Percina (Percidae) in the Kaskaskia River, Illinois. III. Natl. Hist. Surv. Biol. Notes 1970, 70, 1-18.

18. Trautman, M.B. The Fishes of Ohio, 2nd ed.; Ohio State University Press: Columbus, OH, USA, 1981.

19. Smith, P.W. The Fishes of Illinois; University of Illinois Press: Urbana, IL, USA, 1979; pp. 267-268.

20. Bunnell, D.B.; Madenjian, C.P.; Croley, T.E. Long-term trends of bloater (Coregonus hoyi) recruitment in Lake Michigan: Evidence for the effect of sex ratio. Can. J. Fish. Aquat. Sci. 2006, 63, 832-844. [CrossRef] 
21. Balesic, H. Comparative Ecology of Four Species of Darters (Etheostominae) in Lake Dauphin and Its Tributary, the Valley River. Master's Thesis, University of Manitoba, Winnipeg, MB, Canada, 1971.

22. Starnes, W.C. The Ecology and Life History of the Endangered Snail Darter, Percina (Imostoma) tanasi Etnier. Ph.D. Thesis, University of Tennessee, Knoxville, TN, USA, 1977.

23. Haag, W.R.; Warren, M.L., Jr. Seasonal feeding specialization on snails by River Darters (Percina shumardi) with a review of snail feeding by other darter species. Copeia 2006, 4, 604-612. [CrossRef]

24. Kuehne, R.A.; Barbour, W.A. The American Darters; University Press of Kentucky: Lexington, KY, USA, 1983.

25. Becker, G.C. Fishes of Wisconsin; University of Wisconsin Press: Madison, WI, USA, 1983; pp. 912-914.

26. Pfleiger, W.L. A distributional study of Missouri fishes. Univ. Kans. Publ. Mus. Natl. Hist. 1971, 20, $225-570$.

27. Cooper, E.L. Fishes of Pennsylvania and the Northeastern United States; The Pennsylvania State University Press: University Park, PA, USA, 1983; pp. 206-207.

28. Sanders, R.E.; Yoder, C.O. Recent collections and food items of River Darters, Percina shumardi (Percidae), in the Markland Dam Pool of the Ohio River. Ohio J. Sci. 1989, 89, 33-35.

29. Chu, C.; Minns, C.K.; Lester, N.P.; Mandrak, N.E. An updated assessment of human activities, the environment, and freshwater fish biodiversity in Canada. Can. J. Fish. Aquat. Sci. 2014, 72, 135-148. [CrossRef]

(C) 2016 by the authors; licensee MDPI, Basel, Switzerland. This article is an open access article distributed under the terms and conditions of the Creative Commons Attribution (CC-BY) license (http://creativecommons.org/licenses/by/4.0/). 